\title{
Geometrical Meaning of Time and the Theory of Relativity
}

\author{
0. A. Olkhov \\ N. N. Semenov Institute of Chemical Physics of Russian Academy of Sciences, Moscow, Russia \\ Email: oleg.olkhov@rambler.ru
}

How to cite this paper: Olkhov, O.A. (2020) Geometrical Meaning of Time and the Theory of Relativity. Journal of High Energy Physics, Gravitation and Cosmolo$g y, 6,1-8$

https://doi.org/10.4236/jhepgc.2020.61001

Received: September 19, 2019

Accepted: November 8, 2019

Published: November 11, 2019

Copyright $\odot 2020$ by author(s) and Scientific Research Publishing Inc. This work is licensed under the Creative Commons Attribution International License (CC BY 4.0).

http://creativecommons.org/licenses/by/4.0/

\begin{abstract}
New geometrical model of time is suggested where time of body's motion is defined as the length of its trajectories in four-dimension space-time. Within suggested approach periodical motions in clocks correspond to definite length of four-dimension trajectories that is clocks appear to be standards for measurements of length in four-dimension space analogously as hard sticks are standards for measurements of length in three-dimension space. This means that space and time are entities of the same geometrical nature. A suggested interpretation of time leads to necessity of changes in general theory of relativity. These changes are unessential for body's motion in weak gravitational field.
\end{abstract}

\section{Keywords}

Special and General Theory of Relativity, Space-Time, Geometrical Interpretation of Time

\section{Introduction}

This work is a continuation of author's investigations on geometrisation of quantum mechanics [1] [2] [3] [4] [5]. As "geometrisation" we understand the investigation of such models of physical phenomena when these phenomena are expressed through understandable geometrical patterns and notions. We suppose that such model representations play an important role in physical investigations-the known example of use of just geometrical model for obtaining new physical results is the general theory of relativity_GTR (review of literature on geomerization in physics see, e.g., [6]) In this work the new geometrical representation of tine leads to necessity of changes in equations of GTR for motions in gravitational field. Preliminary results are published in [7] [8] [9]. 
To clarify what we mean speaking about "new" geometrical representation of time, we remind shortly about geometrical representation of time within existing special theory of relativity-STR [10] [11] [12] [13]. STR considers space and time as unique space-time continuum which points are named as "events." The set of four numbers $\left(t, x_{2}, x_{2}, x_{3}\right)$ corresponds to each event, where $t$ is the time when this event happened and $x_{2}, x_{2}, x_{3}$ are coordinates of the place of event. This four-dimension space-time continuum is named as "space of events". It represents a so called four-dimension Cartesian space where there is no initial geometry [11]. There only Lorentz transformations are defined on this set of variables connecting space and time coordinates in different inertial frames. Geometrical model of this space was suggested about hundred years ago by Minkowski, and in this model time multiplied by light velocity has a meaning of the fourth coordinate $\left(c t=x_{4}\right)$ in the four-dimension pseudo Euclidean space and the Lorentz transformations appears to be the group of 4-rotations $\mathrm{n}$ this space (since then this space is named as the MInkowski space). This space has mainly methodical value, and it is used in STR for descriptive representation of consequences of the Lorentz transformations. But the formal representation of time by fourth coordinate does not give the opportunity to understand how the readings of clocks correspond to such representation of time. Thus the reason for deep distinction of notions of "space" and "time" remains not clear.

It should be noticed that Feynman was, may be, the first who supposed that space and time are entities of the same nature. He made this suggestion discussing the Lorentz transformation in his book "The Feynman lectures on physics" [14]. When the frame $S^{\prime}$ are moving relative to the frame $S$ with velocity $v$ in direction of the axis $X$, the Lorentz transformations has the form [10] [11] [12] [13]

$$
x^{\prime}=\frac{x-\beta c t}{\sqrt{1-\beta^{2}}}, t^{\prime}=\frac{t-\frac{\beta}{c} x}{\sqrt{1-\beta^{2}}}, y^{\prime}=y, \quad z^{\prime}=z, \quad \beta=\frac{v}{c}
$$

Feinman indicated that in (1) coordinated changes of space coordinates and time in different frames look as coordinated changes of visible body's width and thickness when they are considered from different points of view: from one point the body "has more thickness and less width", from another point body "has more width and less thickness", and this demonstrates the fact that width and thickness expressed the unique, general for body, entity- "the length." In the same way, as Feinman supposed, changes of coordinates and time in (1) describes coordinated changes of notions of the same nature.

The reflection of space of events on the four-dimension space with geometry, different from geometry of Minkowski space (different isomorphism), is suggested in this work. Within this reflection time of motion of macroscopic body (material point) has different meaning and it appears to be the notion of the same nature as the space. 


\section{Geometrical Meaning of Time and New Geometrical Model of the Space of Events}

Let us consider the space of events for a case when event is a displacement of free particle (material point) from the origin of some inertial coordinate system (it is considered as immovable) to the point with coordinates $x_{1}, x_{2}, x_{3}$ for the time $t$. The new isomorphism of such space of events in the four-dimension space with definite geometry is based on following suggestions:

1) Coordinates $x_{1}, x_{2}, x_{3}$ are the particle coordinates in three dimensional affine subspace of four-dimension affine space $x^{1}, x^{2}, x^{3}, x^{4}$

2) Time of the particle displacement at distance $L_{3}$ in the point with coordinates $x_{1}, x_{2}, x_{3}$, multiplied by light velocity, equals to $L_{g}$-the length of the particle straight geodesic trajectory in the above four-dimension affine space

$$
c t=L_{g}
$$

We place here coordinate' indexes upwards to stress the distinction between covariant and contravariant coordinates in affine space [10]. Two dimensional analogies of Minkowski space and suggested space are represented for comparison in Figure 1.

The Lorentz transformations have here, as in Minkowski model, the meaning of transformations transferring the space into itself. The transformations (1) express in STR the invariance of quadratic form

$$
x^{2}+y^{2}+z^{2}=c^{2} t^{2}
$$

respectively to transfer from one inertial frame to another (here $x, y, z$-orthogonal coordinates of the light wave front in three dimensional space, $c$-light velocity in vacuum) [10] [11] [12] [13]. Relation (3) has no meaning for affine space-there is no specific orthonormal frames in affine space [9]. So quadratic form at the left side in (3) does not define the square of any distance in this space. Therefore the geometrical properties of the suggested affine space are defined not by relation (3) for the light wave propagation (as in Minkowski model) but they are defined from relation (analogous by physical meaning) for the limiting velocity of particle's motion, which equals to the light velocity in vacuum. This relation has the form

$$
L_{3}=L_{g}
$$

This relation means, that

$$
\frac{L_{3}}{L_{g}}=\frac{L_{3}}{c t}=\frac{v}{c}=1,
$$
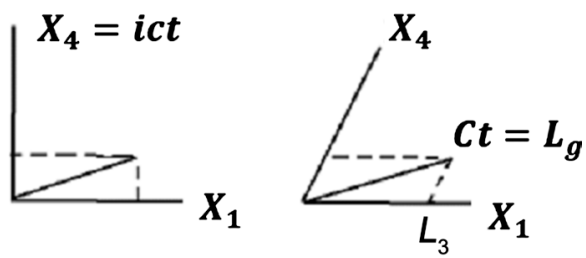

Figure 1. Minkowski space (at left) and affine space. 
that is relation (4) defines the limiting velocity of particle's displacement in three dimensional space (that equals to light velocity). So the Lorentz transformations lead now to invariance of relation (4) for two geometrical characteristic of the suggested affine space and, in this way, these transformations has now the meaning of linear transformations of $L_{3}$ and $L_{g}$, defiing the concrete affine geometry of the space (as in Minkowski model the Lorentz transformations has the meaning of the group of 4-ronations for pseudo Euclidean space). With variables $L_{3}$ and $L_{g}$ the Lorentz transformations (1) has, obviously, the form

$$
L_{3}^{\prime}=\frac{L_{3}-\beta L_{g}}{\sqrt{1-\beta^{2}}}, L_{g}^{\prime}=\frac{L_{g}-\beta L_{3}}{\sqrt{1-\beta^{2}}}, y^{\prime}=y, z^{\prime}=z, \beta=\frac{v}{c} .
$$

Let us stress the main distinctions of suggested model of the space of events from the Minkowski model.

Firstly, the new understanding of time is suggested. Time and space are now considered in physics as different fundamental entities, not reducing to more deep notions and having principally different standards for measurementshard sticks for space and periodical movements (clocks) for time. Because of the standards of space have the dimensionality of length, the notion of space seems intuitively as understandable one whereas the notion of time looks for many people as something mysterious because, mainly, of impossibility to answer the question: "what the clocks show". It is shown in this work that "time" is a notion that does not differs principally from the notion of the "space", because it is defined within suggested approach by the same space characteristic - by the length. And clocks (as hard sticks) appear to be the standards of length for four-dimension geodesics because every periodical movements in clocks corresponds to definite length of corresponding geodesic trajectory-and this fact explains what "really" clocks measure. This is obvious for clocks where periodical movements are movements in gravitational field (for planets motions, for example) because, according to GTR, trajectories in gravitational field are four-dimension geodesics in curved four-dimension space-time [10] [11] [12] [13]. For clocks of another type this is right only under supposition that geodesic trajectories corresponds to motions in any fields.

Secondly, in contrast to Minrowski model surrounding three dimensional space is supposed to be not Euclidean, but affine space. Within such approach the known effects of STR (retarding of time, reduction of length) appear to usual proprieties of affine space, where form and volume of objects are changing under coordinate transformations [10]. At not relativistic velocities three dimensional space may be considered as Euclidean one and four-dimension affine space, considered above, may be considered as four-dimension pseudo Euclidean Minkowski space. This follows from the fact that the Lorentz transformations (1) and (6) (transferring the spaces into itself) reduces both $v / c \rightarrow 0$ (as it should be) to the Galileo transformations

$$
x^{\prime}=x-\beta \tau, t^{\prime}=t, y=y^{\prime}, z=z^{\prime}, \beta=\frac{v}{c} .
$$


Above results (geometrical meaning of time, affine character of the three dimensional space) has not only methodological value. We will show below that suggested geometrical interpretation of time leads to necessity of changes in known equations of motions in gravitational field (equations of motions of GTR).

\section{Geometrical Meaning of Time and General Theory of Relativity}

The geometrical interpretation of time was suggested in previous Section for the free particl's motion, which is for motion in space without of the force fields. Let us consider the particle's motion in gravitational field. As in GTR we suppose that the particle's trajectories in gravitational field are geodesic lines of four-dimension space-time [10] [11] [12] [13]. We suppose in addition that suggested interpretation of time conserves for the particle's motion in gravitational field, that is time of motion, multiplied by light velocity, equals to the length of particle's four-dimension geodesic trajectories. This suggestion looks as natural, if we consider "switching on" of gravitational field as continuous transfer from the "flat" space of events, considered in the previous Section, to the "curved" space-time of GTR.

The suggested geometrical meaning of time leads to necessity of changes in equations of motions of GTR, because, first of all, that in existing theory time enters in above equations as the fourth coordinate of four-dimension space-time whereas the time is defined within suggested approach as the length of the particle's four-dimension trajectories in this space. As in existing GTR we suppose that equations for particle's trajectories in gravitational field are equations for geodesic lines in four-dimension space-time. These equations have in general case the form [10] [11] [12] [13]

$$
\frac{\mathrm{d}^{2} x^{k}}{\mathrm{~d} \tau^{2}}=-\Gamma_{i j}^{k} \frac{\mathrm{d} x^{i}}{\mathrm{~d} \tau} \frac{\mathrm{d} x^{j}}{\mathrm{~d} \tau}, i, j=1,2,3,4
$$

where $\tau$-canonical parameter, $x^{1}, x^{2}, x^{3}, x^{4}$-the particle's coordinates, $\Gamma_{i j}^{k}-$ connectedness of the "curved" by field space [10] [11] [12] [13].

New equations have formally the same form as the existing equations, but they principally differs from them because the time variable enters in the new equations in different way. In existing theory time, multiplied by light velocity, enters to the left side of Equation (8) as the fourth coordinate $x^{4}$, but within suggested interpretation of time, we suppose that time of the particle's motion, multiplied by light velocity, is defined to be the length of the particle's geodesic trajectory. Choosing the length of geodesic trajectory as canonic parameter, we obtain, that time enters to the right side of Equation (8) instead of canonic parameter according to the relation $\tau=c t$. After this substitution in (8) the new equations of motion take the form

$$
\frac{\mathrm{d}^{2} x^{k}}{\mathrm{~d} t^{2}}=-\Gamma_{i j}^{k} \frac{\mathrm{d} x^{i}}{\mathrm{~d} t} \frac{\mathrm{d} x^{j}}{\mathrm{~d} t}, i, j=1,2,3,4
$$


Thus, equations of the particle's motion in gravitational field (9) differs within suggested interpretation of time from corresponding equations of GRT (8), where at the left side $x^{4}=c t$.

Let us show that new equations turn in the equations of existing theory for motion in weak gravitational field at $v / c \rightarrow 0$ and therefore are in agreement with experiment. We showed in previous Section that at not relativistic velocities three-dimension space may be considered as Euclidean one and four-dimension affine space, considered above, may be considered as four-dimension pseudo Euclidean Minkowski space. Therefore it is natural to suppose, as in existing theory, that "curved" by field space may be considered within above approximation as the pseudo Riemannian space. Then, as in existing theory, connectedness $\Gamma_{i j}^{k}$ in (9) may be considered as connectedness of pseudo Riemannian space.

We will show now that for motions in weak gravitational field the time enters in equations of motion in the same way as in existing theory, namely, as the fourth coordinate. Without gravitational field, when the space-time curvature equals zero, the metric of four-dimension pseudo Riemannian space equals to the metric of four-dimension pseudo Euclidean space that is has the form [10] [11] [12] [13]

$$
\mathrm{d} s^{2}=\mathrm{d} x^{1^{2}}+\mathrm{d} x^{2^{2}}+\mathrm{d} x^{3^{2}}-\mathrm{d} x^{4^{2}} .
$$

Fur the same space this metric can be rewritten with another signature as [10]

$$
\mathrm{d} s^{2}=-\mathrm{d} x^{1^{2}}-\mathrm{d} x^{2^{2}}-\mathrm{d} x^{3^{2}}+\mathrm{d} x^{4^{2}} .
$$

Considering $\mathrm{d} s$ as the element of length of geodesic line and taking in mind the geometrical representation of time (5), we have from (11)

$$
\mathrm{d} x^{4}=c \mathrm{~d} t \sqrt{1+\frac{v_{1}^{2}}{c^{2}}+\frac{v_{2}^{2}}{c^{2}}+\frac{v_{3}^{2}}{c^{2}}},
$$

where

$$
v_{i}=\frac{\mathrm{d} x^{i}}{\mathrm{~d} t}, i=1,2,3
$$

It follows from (12) \& (13), that at $v / c \rightarrow 0$ we can write with accuracy $(v / c)^{2}$

$$
\mathrm{d} x^{4}=c \mathrm{~d} t,
$$

This means, that change of time, multiplied by light velocity, equals with above accuracy to change of the forth particle's coordinate, as it is supposed for equations off motion in the existing theory. Thus, at $v / c \rightarrow 0$ new equations of motion in gravitational field (9) go over into equations of existing theory, that is they lead to the known results coinciding with experiment (Newtonian approximation, correction of the Mercury orbit).

New geometrical representation of time and possible changes of GTR for strong gravitational field may be important, first of all, at consideration of some problems in Cosmology (see, e.g., [15] [16]). Investigation of Equation (9) in general case and investigation of possible changes in GTR and Cosmology, connecting with these new equations, need special consideration and they are not considered here. 


\section{Conclusion}

The geometrical interpretation of time is suggested as the length of the particle's geodesic trajectory in the four-dimension space-time. This new interpretation of time leads to changes in the known equations of general theory of relativity for motions in strong gravitational field. As time enters also in equations of motion of charged particles in electromagnetic field, the suggested interpretation of time indicates the possibility of geometrical interpretation of electromagnetic field, as it was believed by Einstein.

\section{Funding}

This work was carried out in the framework of State Assignment of the Ministry of Science and Higher Education of the Russian Federation (project 0082-20190017, registration code AAAA-A19-119010990034-5).

\section{Conflicts of Interest}

The authors declare no conflicts of interest regarding the publication of this paper.

\section{References}

[1] Olkhov, O.A. (2008) Geometrisation of Quantum Mechanics. Journal of Physics Conference Series, 67, Article ID: 012037. https://doi.org/10.1088/1742-6596/67/1/012037

[2] Olkhov, O.A. (2008) On the Possibility of Topological Interpretation of Quantum Mechanics. http://arXiv.org/abs/0802.2269

[3] Olkhov, O.A. (2008) Geometrisation of Classical Wave Fields. AIP Conferences Proceedings, 962, 316-321. https://doi.org/10.1063/1.2827325

[4] Olkhov, O.A. (2014) Geometrical Approach in the Atomic Spectra Theory. The Helium Atom. Russian Journal of Physical Chemistry B, 8, 30-42. https://doi.org/10.1134/S199079311401014X

[5] Olkhov, O.A. (2014) Geometrical Approach in Atomic Physics. Atoms of Hydrogen and Helium. American Journal of Physics and Applications, 2, 108-112. https://doi.org/10.11648/j.ajpa.20140205.12

[6] Vladimirov, Yu.S. (2005) Geometrophysics. Binom, Moscow. (In Russian)

[7] Olkhov, O.A. (2017) On Possibility of Geometrical Interpretation of Time. Journal of High Energy Physics, Gravitation and Cosmology, 3, 173-177. https://doi.org/10.4236/jhepgc.2017.32018

[8] Olkhov, O.A. (2018) The Geometrical Meaning of Time and the Limiting Speed of Signal Propagation. Russian Journal of Physical Chemistry B, 12, 333-336. https://doi.org/10.1134/S1990793118050202

[9] Olkhov, O.A. (2019) Geometrical Meaning of Time and New Formulation of the Theory of Relativity. Abstracts of XXI International Scientific Conference Physical Interpretation of Relativity Theory, Moscow, 89.

[10] Rashevski, P.K. (1967) Riemann Geometry and Tensor Analisis. Nauka, Moscow. (In Russian)

[11] Dubrovin, B.A., Fomenko, A.T. and Novikov, S.P. (1985) Modern Geometry-Methods 
and Applications. Springer, Berlin, Vol. 1, 1-4, Vol. 2, 31.

https://doi.org/10.1007/978-1-4612-1100-6

[12] Pauli, W. (1958) Theory of Relativity. Pergamon Press, Oxford.

[13] Landau, L.D. and Lifchitz, E.M. (1975) Course of Theoretical Physics, Volume 2. The Classical Theory of Fields. Butterworth-Heinemann, Oxford, Ch. 1, 6, Ch. 11.

[14] Feynman, R., Leighton, R. and Sands, M. (1963) The Feynman Lectures on Physics. Addison-Wesley Publishing Company, Boston.

[15] Mukhanov, V. (2005) Physical Foundations of Cosmology. Cambridge University Press, Cambridge. https://doi.org/10.1017/CBO9780511790553

[16] Khlopov, M. (2012) Fundamentals of Cosmic Particle Physics. Cambridge International Science Publishing, Cambridge. https://doi.org/10.1007/978-1-907343-72-8 